\title{
ANTIFUNGAL PEROXYKETAL ACIDS FROM AN OKINAWAN MARINE SPONGE OF PLAKORTIS SP.
}

Motomasa KOBAYASHI, Kazunari KONDO, and Isao KITAGAWA*

Faculty of Pharmaceutical Sciences, Osaka University,

Yamada-oka 1-6, Suita, Osaka 565, Japan

Antifungal peroxyketal acids and their methyl esters, the peroxyplakoric acids $A_{1}$ methyl ester (1), A2 methyl ester (2), A3 methyl ester (3), B1 methyl ester (4), and B 3 methyl ester (5), were separated from an Okinawan marine sponge of Plakortis sp. The absolute stereostructures $\mathbf{1} \sim \mathbf{5}$ have been elucidated on the basis of chemical and physicochemical evidence.

KEYWORDS peroxyplakoric acid $A_{1}$ methyl ester; peroxyplakoric acid $B_{1}$ methyl ester; marine sponge; Plakortis sp; antifungal activity; peroxyketal

A number of cyclic peroxides have been isolated from marine organisms; in particular, marine sponges of the genus Plakortis are rich sources of cyclic peroxides and peroxyketals. ${ }^{1}$ ) These peroxy compounds generally exhibit antimicrobial, ichthyotoxic, and cytotoxic activities. During the course of our investigations in search of new biologically active substances from marine organisms, ${ }^{2}$ ) we have isolated antifungal peroxyketal acids and their methyl esters, peroxyplakoric acids A1 methyl ester (1), A2 methyl ester (2), A3 methyl ester (3), B1 methyl ester (4), and B3 methyl ester (5), from an Okinawan marine sponge of Plakortis sp. 3 ) This paper communicates the absolute stereostructure elucidation of these peroxyketal acids.

The AcOEt soluble portion $(6.85 \mathrm{~g})$, separated from the acetone extract of the fresh titled sponge (1 kg, collected in July at Zamami Island, Okinawa Prefecture), was subjected to repeated $\mathrm{SiO}_{2}$ column chromatography to provide a mixture of antifungal peroxyketal acids $(0.64 \%$ from the AcOEt soluble portion). This peroxyketal acid mixture showed strong antifungal activity [30 $\mathrm{mm}$ diameter growth inhibition for Candida tropicals at $40 \mu \mathrm{g} / \mathrm{disk}(\phi=8 \mathrm{~mm})]$. The peroxyketal acids were so unstable as to be isolated to each component either by TLC or by HPLC. Thus, the peroxyketal acid mixture was treated with diazomethane to yield methyl esters, which were then separated by $\mathrm{SiO}_{2}$ column and HPLC to provide five compounds: peroxyplakoric acids A 1 methyl ester (1) $\sim$ B3 methyl ester (5). Larger amounts of these peroxyketal methyl esters $1 \sim 5$ were obtained from the initial AcOEt soluble portion of this sponge in $1.8,0.8,3.4,1$, and $0.8 \%$ yields, respectively.

Peroxyplakoric acid $A_{1}$ methyl ester (1) was obtained as an amorphous solid: $[\alpha]_{D}-164^{\circ}$ $\left(\mathrm{CHCl}_{3}\right)$; UV $\lambda \max (\mathrm{MeOH}): 235 \mathrm{~nm}(\varepsilon=22000)$; IR $(\mathrm{KBr}): 1740,1063,965 \mathrm{~cm}^{-1}$. The FAB-MS of 1 showed a quasi-molecular ion peak at $\mathrm{m} / \mathrm{z} 363(\mathrm{M}+\mathrm{Na})^{+}$, and the molecular formula has been determined as $\mathrm{C}_{19} \mathrm{H}_{32} \mathrm{O}_{5}$ by $\mathrm{HR}$ FAB-MS and NMR analysis. All proton and carbon signals in the NMR spectra of 1 were assigned as given in Tables I and II on the bases of H-H COSY, C-H COSY, and COLOC experiments, and the plain structure of 1 has been determined. Thus, the following COLOC correlations were essential: cross peaks observed between $\mathrm{C}-1$ and $\mathrm{H}-16$ and carbomethoxyl protons; between $\mathrm{C}-6$ and $\mathrm{H}-5, \mathrm{H}-7$ and methoxyl protons; between $\mathrm{C}-12$ and $\mathrm{H}-11$ and $\mathrm{H}$ 17. The relative stereostructure of the peroxide ring in 1 has been elucidated on the basis of the NOESY correlation: e.g. cross peaks observed between $\mathrm{H}-4 \beta$ and methoxyl protons; between $\mathrm{H}-3 \alpha$ and $\mathrm{H}-4 \alpha, \mathrm{H}-5 \alpha$. The relative stereostructures of peroxyplakoric acids A2 methyl ester $(2)^{4)}$ and A3 methyl ester $\left.(3)^{5}\right)$ have been elucidated in the same manner. ${ }^{6}$ )

The plain structures of peroxyplakoric acids $B_{1}$ methyl ester (4)7) and $B_{3}$ methyl ester (5) 8 ) have also been calculated from the 2D NMR experiments. Furthermore, the NOESY correlations (e.g. cross peaks between $\mathrm{H}-4 \beta$ and methoxyl protons; between $\mathrm{H}-2$ and $\mathrm{H}-4 \alpha, \mathrm{H}-5 \alpha$; between $\mathrm{H}-$ 16 and $H-4 \alpha$ ) in 4 and 5 have led to the relative configuration of the respective peroxide rings. In order to determine the absolute stereostructures $\mathbf{1} \sim \mathbf{5}$, the following conversions have been carried out. First, peroxyplakoric acid $\mathrm{A}_{3}$ methyl ester (3) was treated with $\mathrm{LiAlH}(t-\mathrm{BuO}) 3$ to furnish the peroxyketal alcohol 6, which was then treated with (+)- and (-)-methoxytrifluoromethylphenylacetic acid (MTPA) and dicyclohexylcarbodiimide (DCC) in the presence of dimethylaminopyridine (DMAP) to provide the (+)-MTPA ester 7 $\mathbf{a}^{9}$ ) and the (-)-MTPA ester 
7b $\mathbf{b}, 9)$ respectively. The $\mathrm{C}-1$ methylene proton signals of $7 \mathbf{a}$ were observed as a pair of doubledoublets at $\delta 4.18(\mathrm{dd}, J=11,6.5)$ and $\delta 4.37(\mathrm{dd}, J=11,5.5)$, while those of $7 \mathrm{~b}$ were observed as a doublet at $\delta 4.26(2 \mathrm{H}, \mathrm{d}, J=6)$. Consequently, the $2 \mathrm{R}$ configuration of 3 has been confirmed. $\left.{ }^{10}\right)$ On the other hand, $\operatorname{LiAlH}(t-\mathrm{BuO}) 3$ treatment of peroxyplakoric acid B3 methyl ester (5) furnished the other peroxyketal alcohol 8, which was again esterified with (+)- and (-)-MTPA as above to confirm the $2 \mathrm{R}$ configuration ${ }^{10)}: \delta 4.42\left(\mathrm{dd}, J=11,5.5, \mathrm{H}_{\mathrm{a}}-1\right)$ and $\delta 4.50$ (dd, $J=11,3, \mathrm{H}_{\mathrm{b}}$ 1) in (+)-MTPA ester 9a9); $\delta 4.43\left(\mathrm{~s}, \mathrm{H}_{\mathrm{a}}-1\right)$ and $\delta 4.45\left(\mathrm{~d}, J=2.5, \mathrm{H}_{\mathrm{b}}-1\right)$ in (-)-MTPA ester $\left.\left.\left.9 \mathrm{~b}^{9}\right)\right] .{ }^{11}\right)$

Next, peroxyplakoric acid A3 methyl ester (3) was subjected to catalytic hydrogenation over $10 \% \mathrm{Pd} / \mathrm{C}$ to furnish the 3-hydroxy-6-keto acid methyl ester $10\left([\alpha] \mathrm{D}-9^{\circ}\left(\mathrm{CHCl}_{3}\right)\right)$, which was further treated with (+)- and (-)-MTPA and DCC in the presence of DMAP to provide the (+)MTPA ester 11 $\mathbf{a}^{12)}$ and the (-)-MTPA ester 11 $\mathbf{b}^{12)}$, respectively. In the ${ }^{1} \mathrm{H}$ NMR spectra of $11 \mathrm{a}$ and $11 \mathrm{~b}$, due to the anisotropic effect of the phenyl ring, the positive $\Delta \delta[\delta(-)-\delta(+)]$ values for the signals of protons of carbomethoxyl $(+0.05)$ and protons at $\mathrm{C}-2(+0.03)$ and $\mathrm{C}-16(+0.04)$ were observed, while the negative $\Delta \delta$ values for the signals of protons at C-5 (-0.15) and C-7 (-0.09) were observed. Thus, the absolute configuration at $\mathrm{C}-3$ of 3 has been shown to be S. ${ }^{13)}$ On the other hand, catalytic hydrogenation over $10 \% \mathrm{Pd} / \mathrm{C}$ of 5 furnished the other 3-hydroxy-6-keto acid methyl ester $12\left([\alpha] \mathrm{D}-4^{\circ}\left(\mathrm{CHCl}_{3}\right)\right)$, which was again converted to the (+)-MTPA ester 13a $\mathbf{a}^{14)}$ and the (-)-MTPA ester 13b.14) In a similar manner, the absolute configuration at C-3

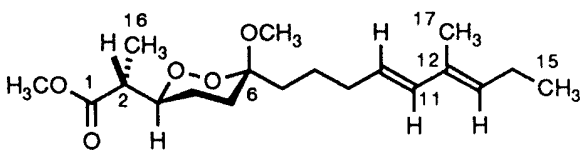

peroxyplakoric acid $A_{1}$ methyl ester (1)<smiles>CC/C=C\C=C\CCCC1(C)C[C@H](OC)[C@@H]([C@@H](C)C(=O)OC)O1</smiles>

peroxyplakoric acid $A_{2}$ methyl ester (2)<smiles>CC/C=C/C=C/CCCC1(C)C[C@H](OC)[C@H](C)OO1</smiles>

peroxyplakoric acid $A_{3}$ methyl ester (3)<smiles>CCCCCCCCCC(=O)CC[C@H](O)[C@H](C)C(=O)OC</smiles><smiles>CC/C=C\C=C\CCCC1(OC)CC[C@H]([C@@H](C)C(=O)OC)OO1</smiles>

peroxyplakoric acid $B_{1}$ methyl ester (4)<smiles>CC/C=C/C=C/CCCC1(OC)CO[C@H]([C@@H](C)C(=O)OC)CO1</smiles>

peroxyplakoric acid $B_{3}$ methyl ester (5)
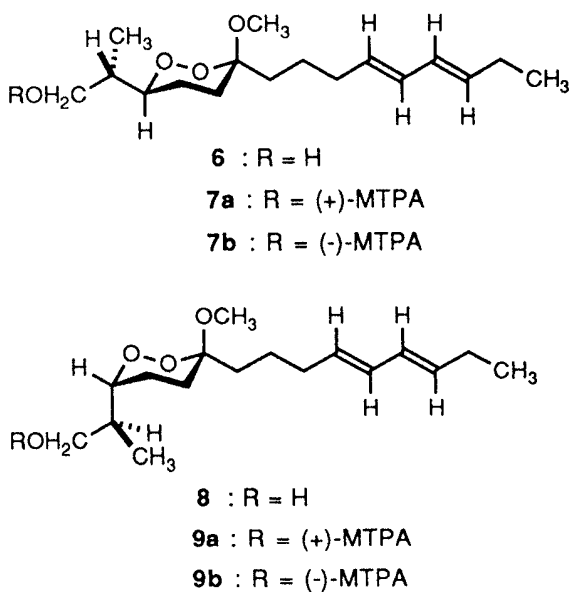

Table I. ${ }^{1} \mathrm{H}$ NMR Data for Peroxyplakoric Acids $A_{1}$ Methyl Ester (1) $\sim B_{3}$ Methyl Ester (5) (at $500 \mathrm{MHz}$ in $\mathrm{CDCl}_{3}, J$ Values in $\mathrm{Hz}$ )

\begin{tabular}{|c|c|c|c|c|c|}
\hline Proton(s) at & 1 & 2 & 3 & 4 & 5 \\
\hline 2 & $2.51(\mathrm{dq}, 7.5,7.5)$ & $2.51(\mathrm{dq}, 7,7)$ & $2.49(\mathrm{dq}, 7,7)$ & $3.07(\mathrm{dq}, 9.5,7)$ & $3.07(\mathrm{dq}, 9.5,7)$ \\
\hline 3 & $4.20(\mathrm{ddd}, 10.5,8,2)$ & $4.20(\mathrm{ddd}, 10.5,8,2)$ & $4.18(\mathrm{ddd}, 10.5,8,2)$ & $4.20(\mathrm{ddd}, 10,6,6)$ & $4.21(\mathrm{ddd}, 9.5,5.5,5.5)$ \\
\hline $4-\alpha$ & $1.52(\mathrm{~m})$ & $1.52(\mathrm{~m})$ & $1.50(\mathrm{~m})$ & $1.42(\mathrm{~m})$ & $1.45(\mathrm{~m})$ \\
\hline $4-\beta$ & $1.85(\mathrm{~m})$ & $1.85(\mathrm{~m})$ & $1.84(\mathrm{~m})$ & $2.10(\mathrm{~m})$ & $2.10(\mathrm{~m})$ \\
\hline $5-\alpha$ & $1.90(\mathrm{~m})$ & $1.90(\mathrm{~m})$ & $1.88(\mathrm{~m})$ & $1.67(\mathrm{~m})$ & $1.65(\mathrm{~m})$ \\
\hline $5-\beta$ & $1.63(\mathrm{~m})$ & $1.63(\mathrm{~m})$ & $1.63(\mathrm{~m})$ & $1.75(\mathrm{~m})$ & $1.75(\mathrm{~m})$ \\
\hline 7 & $1.63(\mathrm{~m}), 1.68(\mathrm{~m})$ & $1.63(\mathrm{~m}), 1.68(\mathrm{~m})$ & $1.63(2 \mathrm{H}, \mathrm{m})$ & $1.63(2 \mathrm{H}, \mathrm{m})$ & $1.65(2 \mathrm{H}, \mathrm{m})$ \\
\hline 8 & $1.35(\mathrm{~m}), 1.45(\mathrm{~m})$ & $1.35(\mathrm{~m}), 1.45(\mathrm{~m})$ & $1.32(\mathrm{~m}), 1.40(\mathrm{~m})$ & $1.42(2 \mathrm{H}, \mathrm{m})$ & $1.45(2 \mathrm{H}, \mathrm{m})$ \\
\hline$\stackrel{0}{9}$ & $2.10(\mathrm{~m})$ & $2.10(\mathrm{~m})$ & $2.10(\mathrm{~m})$ & $2.10(\mathrm{~m})$ & $2.10(\mathrm{~m})$ \\
\hline 10 & $5.51(\mathrm{~m})$ & $5.62(\mathrm{~m})$ & $5.61(\mathrm{dt}, 13.5,6.5)$ & $5.51(\mathrm{~m})$ & $5.64(\mathrm{dt}, 13.5,6.5)$ \\
\hline 11 & $6.05(\mathrm{~d}, 15.5)$ & $6.44(\mathrm{~d}, 15.5)$ & $5.99(\mathrm{~m})$ & $6.06(\mathrm{~d}, 15.5)$ & $6.01(\mathrm{~m})$ \\
\hline 12 & - & 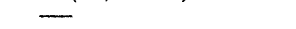 & $5.99(\mathrm{~m})$ & का & $6.01(\mathrm{~m})$ \\
\hline 13 & $5.37(t, 7)$ & $5.27(t, 7)$ & $5.51(\mathrm{dt}, 14,6.5)$ & $5.38(t, 7)$ & $5.53(\mathrm{dt}, 14,6.5)$ \\
\hline 14 & $2.15(\mathrm{~m})$ & $2.15(\mathrm{~m})$ & $2.10(\mathrm{~m})$ & $2.15(\mathrm{~m})$ & $2.10(\mathrm{~m})$ \\
\hline 15 & $0.98(t, 7.5)$ & $0.98(t, 7)$ & $0.99(t, 7.5)$ & $0.98(t, 7.5)$ & $1.01(t, 7.5)$ \\
\hline 16 & $1.25(\mathrm{~d}, 7)$ & $1.25(\mathrm{~d}, 7)$ & $1.23(\mathrm{~d}, 7)$ & $1.14(\mathrm{~d}, 7)$ & $1.15(\mathrm{~d}, 7)$ \\
\hline 17 & $1.71(\mathrm{~s})$ & $1.78(\mathrm{~s})$ & - & $1.71(\mathrm{~s})$ & 1. \\
\hline $1-\mathrm{OCH}_{3}$ & $3.70(\mathrm{~s})$ & $3.70(\mathrm{~s})$ & $3.68(\mathrm{~s})$ & $3.72(\mathrm{~s})$ & $3.73(\mathrm{~s})$ \\
\hline $6-\mathrm{OCH}_{3}$ & $3.26(\mathrm{~s})$ & $3.26(\mathrm{~s})$ & $3.24(\mathrm{~s})$ & $3.27(\mathrm{~s})$ & $3.28(\mathrm{~s})$ \\
\hline
\end{tabular}




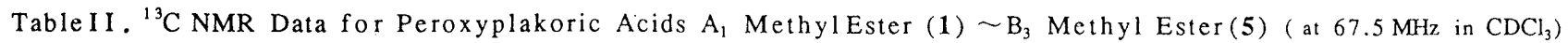

\begin{tabular}{|c|c|c|c|c|c|}
\hline Carbon at & 1 & 2 & 3 & 4 & 5 \\
\hline $\begin{array}{l}1 \\
2 \\
3 \\
4 \\
5 \\
6 \\
7 \\
8 \\
9 \\
10 \\
111 \\
12 \\
13 \\
14 \\
15 \\
16 \\
17 \\
1-\mathrm{OCH}_{3} \\
6-\mathrm{OCH}_{3} \\
\end{array}$ & $\begin{array}{r}173.9(\mathrm{~s}) \\
43.3(\mathrm{~d}) \\
81.0(\mathrm{~d}) \\
23.2(\mathrm{t}) \\
30.4(\mathrm{t}) \\
102.7(\mathrm{~s}) \\
32.1(\mathrm{t}) \\
22.9(\mathrm{t}) \\
32.7(\mathrm{t}) \\
126.2(\mathrm{~d}) \\
135.5(\mathrm{~d}) \\
132.7(\mathrm{~s}) \\
132.7(\mathrm{~d}) \\
21.3(\mathrm{t}) \\
14.1(\mathrm{q}) \\
13.2(\mathrm{q}) \\
12.2(\mathrm{q}) \\
51.8(\mathrm{q}) \\
48.4(\mathrm{q})\end{array}$ & $\begin{array}{r}173.9(\mathrm{~s}) \\
43.3(\mathrm{~d}) \\
81.1(\mathrm{~d}) \\
23.2(\mathrm{t}) \\
30.4(\mathrm{t}) \\
102.7(\mathrm{~s}) \\
32.1(\mathrm{t}) \\
22.9(\mathrm{t}) \\
33.2(\mathrm{t}) \\
127.9(\mathrm{~d}) \\
129.3(\mathrm{~d}) \\
131.0(\mathrm{~s}) \\
131.0(\mathrm{~d}) \\
20.6(\mathrm{t}) \\
14.4(\mathrm{q}) \\
13.2(\mathrm{q}) \\
20.4(\mathrm{q}) \\
51.8(\mathrm{q}) \\
48.4(\mathrm{q})\end{array}$ & $\begin{array}{r}173.8(\mathrm{~s}) \\
43.3(\mathrm{~d}) \\
81.0(\mathrm{~d}) \\
23.2(\mathrm{t}) \\
30.4(\mathrm{t}) \\
102.6(\mathrm{~s}) \\
32.0(\mathrm{t}) \\
22.6(\mathrm{t}) \\
32.4(\mathrm{t}) \\
134.4(\mathrm{~d}) \\
129.1(\mathrm{~d}) \\
131.1(\mathrm{~d}) \\
130.9(\mathrm{~d}) \\
25.5(\mathrm{t}) \\
13.5(\mathrm{q}) \\
13.1(\mathrm{q}) \\
51.8(\mathrm{q}) \\
48.4(\mathrm{q})\end{array}$ & $\begin{array}{r}175.1(\mathrm{~s}) \\
41.6(\mathrm{~d}) \\
81.0(\mathrm{~d}) \\
20.4(\mathrm{t}) \\
26.5(\mathrm{t}) \\
104.5(\mathrm{~s}) \\
30.9(\mathrm{t}) \\
22.8(\mathrm{t}) \\
32.7(\mathrm{t}) \\
126.2(\mathrm{~d}) \\
135.6(\mathrm{~d}) \\
132.8(\mathrm{~s}) \\
132.8(\mathrm{~d}) \\
21.3(\mathrm{t}) \\
14.1(\mathrm{q}) \\
13.7(\mathrm{q}) \\
12.2(\mathrm{q}) \\
51.8(\mathrm{q}) \\
48.9(\mathrm{q})\end{array}$ & $\begin{array}{r}175.2(\mathrm{~s}) \\
41.6(\mathrm{~d}) \\
81.0(\mathrm{~d}) \\
20.4(\mathrm{t}) \\
26.5(\mathrm{t}) \\
104.5(\mathrm{~s}) \\
30.9(\mathrm{t}) \\
22.5(\mathrm{t}) \\
32.5(\mathrm{t}) \\
134.6(\mathrm{~d}) \\
129.1(\mathrm{~d}) \\
131.2(\mathrm{~d}) \\
130.9(\mathrm{~d}) \\
25.6(\mathrm{t}) \\
13.8(\mathrm{q}) \\
13.6(\mathrm{q}) \\
51.9(\mathrm{q}) \\
48.8(\mathrm{q})\end{array}$ \\
\hline
\end{tabular}

of 5 has been shown to be $\mathrm{R}$ by means of NMR analysis [ $\Delta \delta$ values for the protons of carbomethoxyl $(-0.04)$ and protons at $\mathrm{C}-2(-0.01), \mathrm{C}-16(-0.04)$, and $\mathrm{C}-7(+0.08)]$. Consequently, the absolute stereostructures of peroxyplakoric acids $\mathrm{A}_{1}$ methyl ester (1), A 2 methyl ester (2), $\mathrm{A}_{3}$ methyl ester (3), $B_{1}$ methyl ester (4), and $B_{3}$ methyl ester (5) have been determined as shown.

\section{ACKNOWLEDGEMENT} Japan for financial support.
The authors are grateful to the Ministry of Education, Science, and Culture of

\section{REFERENCES AND NOTES}

1) a) D. B. Stierle and D. J. Faulkner, J. Org. Chem., 45, 3396 (1980); b) D. W. Phillipson and K. L. Rinehart, Jr., J. Am. Chem. Soc., 105, 7735 (1983); c) S. Sakemi, T. Higa, U. Anthoni, and C. Christophersen, Tetrahedron, 43, 263 (1987); d) T. Murayama, Y. Ohizumi, H. Nakamura, T. Sasaki, and J. Kobayashi, Experientia, 45, 898 (1989); e) S. P. Gunasekera, M. Gunasekera, G. P. Gunawardana, P. McCarthy, and N. Burres, J. Nat. Prod., 53, 669 (1990); f) B. S. Davidson, J. Org. Chem., 56, 6722 (1991); g) J. Kobayashi, S. Takeuchi, M. Ishibashi, H. Shigemori, and T. Sasaki, Tetrahedron Lett., 33, 2579 (1992).

2) The preceding paper: M. Kobayashi, S. Aoki, H. Sakai, N. Kihara, T. Sasaki, and I. Kitłgawa, Chem. Pharm. Bull., 41, 989 (1993).

3) These peroxyketal acids were called plakortic acids $A \sim B$ in our oral presentation (The 113th Annual Meeting of the Pharmaceutical Society of Japan, April 1993. Abstract Paper Vol. 2, p. 159). However, we then noticed that plakortic acid was already used to name a marine spongean product. ${ }^{\mathrm{b}}$ ) We have re-named our peroxyketal acids peroxyplakoric acid.

4) 2: $[\alpha]_{\mathrm{D}}-163^{\circ}\left(\mathrm{CHCl}_{3}\right) ; \mathrm{UV}(\mathrm{MeOH}): 237 \mathrm{~nm}(\varepsilon=20000) ; \mathrm{IR}(\mathrm{KBr}): 1732,1171,1055,964 \mathrm{~cm}^{-1}$; FAB-MS: $\mathrm{m} / \mathrm{z}$ $363(\mathrm{M}+\mathrm{Na})^{+}\left(\mathrm{C}_{19} \mathrm{H}_{32} \mathrm{O} 5 \mathrm{Na}\right.$ by HR FAB-MS $)$.

5) 3: $[\alpha]_{\mathrm{D}}-167^{\circ}\left(\mathrm{CHCl}_{3}\right) ; \mathrm{UV}(\mathrm{MeOH}): 230 \mathrm{~nm}(\varepsilon=21000) ;$ IR $(\mathrm{KBr}): 1740,1197,1063,990 \mathrm{~cm}^{-1}$; FAB-MS: $\mathrm{m} / \mathrm{z}$ $349(\mathrm{M}+\mathrm{Na})^{+}\left(\mathrm{C}_{18} \mathrm{H}_{30} \mathrm{O}{ }_{5} \mathrm{Na}\right.$ by HR FAB-MS $)$.

6) The geometry of $\Delta^{12}$ in 1,2 , and 4 have been substantiated from the NOESY correlations between $H-11$ and $\mathrm{H}-13$ and between $\mathrm{H}-17$ and $\mathrm{H}-14$ or $\mathrm{H}-13$.

7) 4: $[\alpha]_{\mathrm{D}}-197^{\circ}\left(\mathrm{CHCl}_{3}\right) ; \mathrm{UV}(\mathrm{MeOH}): 235 \mathrm{~nm}(\varepsilon=22000) ;$ IR $(\mathrm{KBr}): 1742,1169,1076,965 \mathrm{~cm}^{-1}$; FAB-MS: $\mathrm{m} / \mathrm{z}$ $363(\mathrm{M}+\mathrm{Na})^{+}\left(\mathrm{C}_{19} \mathrm{H}_{32} \mathrm{O}_{5} \mathrm{Na}\right.$ by HR FAB-MS $)$.

8) 5: $[\alpha] \mathrm{D}-191^{\circ}\left(\mathrm{CHCl}_{3}\right)$; UV (MeOH): $230 \mathrm{~nm}(\varepsilon=21000) ; \mathrm{IR}(\mathrm{KBr}): 1742,1171,1074,990 \mathrm{~cm}^{-1}$; FAB-MS: $\mathrm{m} / \mathrm{z}$ $349(\mathrm{M}+\mathrm{Na})^{+}\left(\mathrm{C}_{18} \mathrm{H}_{30} \mathrm{O} 5 \mathrm{Na}\right.$ by HR FAB-MS $)$.

9) $7 \mathbf{a}, 7 \mathbf{b}, 9 \mathbf{a}$, and $9 \mathbf{b}: \mathrm{m} / z 537(\mathrm{M}+\mathrm{Na})^{+}\left(\mathrm{C}_{2} 7 \mathrm{H}_{37} \mathrm{O}_{6} \mathrm{~F} 3 \mathrm{Na}\right.$ by HR FAB-MS $)$.

10) F. Yasuhara, S. Yamaguchi, R. Kasai, and O. Tanaka, Tetrahedron Lett., 27, 4033 (1986).

11) Peroxyplakoric acid $B_{1}$ methyl ester (4) and a mixture of peroxyplakoric acids $A_{1}$ methyl ester (1) and $\mathrm{A}_{2}$ methyl ester (2) were also converted to the respective C-1 MTPA esters and analyzed in the same manner to result in the same conclusion as observed for $\mathbf{5}$ and $\mathbf{3}$, respectively.

12) 11a, 11b: $m / z 517(\mathrm{M}+\mathrm{H})^{+}\left(\mathrm{C}_{2} 7 \mathrm{H}_{40} \mathrm{O}_{6} \mathrm{~F}_{3}\right.$ by HR FAB-MS).

13) a) T. Kusumi, I. Ohtani, M. Inoue, and H. Kakisawa, Tetrahedron Lett., 29, 4731 (1988); b) S. Takano, M. Takahashi, M. Yanase, Y. Sekiguchi, and K. Ogasawara, Chem. Lett., 1988, 1827.

14) 13a, 13b: $\mathrm{m} / z 539(\mathrm{M}+\mathrm{Na})^{+}\left(\mathrm{C}_{27} \mathrm{H}_{39} \mathrm{O}_{6} \mathrm{~F}_{3} \mathrm{Na}\right.$ by HR FAB-MS $)$. 